\title{
Buprenorphine Microdose Induction for the Management of Prescription Opioid Dependence
}

\author{
Jonathan L. Robbins, MD, MS, Honora Englander, MD, and Jessica Gregg, MD, PhD
}

Prescription opioid dependence remains a major source of morbidity and mortality in the United States. Patients previously on high-dose opioids may poorly tolerate opioid tapers. Current guidelines support the use of buprenorphine therapy in opioid-tapering protocols, even among patients without a diagnosis of opioid use disorder. Buprenorphine microinduction protocols can be used to transition patients to buprenorphine therapy without opioid withdrawal. From November 2019 to April 2020, we transitioned 8 patients on high-dose prescribed opioids for pain to sublingual buprenorphine-naloxone using a microdose protocol without any evidence of precipitated withdrawal. Six of these patients remain on buprenorphine-naloxone and report improved analgesia. Because of its simplicity, the buprenorphine microinduction protocol can be easily adapted for telemedicine and may help to prevent unnecessary clinic visits and opioid-related admissions in the setting of social distancing regulations during the coronavirus 2019 pandemic. (J Am Board Fam Med 2021;34:S141-S146.)

Keywords: Buprenorphine Naloxone, COVID-19, Opioid Addiction, Pain, Pandemics, Telemedicine

\section{Background}

Prescription opioid dependence remains a significant source of morbidity and mortality, with nearly 15,000 deaths attributable to prescription opioid overdose in 2018. ${ }^{1-3}$ Shared decision making regarding the risks and benefits of chronic opioid therapy and voluntary opioid tapering can help to mitigate opioid side effects. ${ }^{4,5}$ However, rapid or forced opioid tapering can destabilize patients and lead to opioid withdrawal and a loss of function, and some patients struggle with slow tapers. ${ }^{6,7}$

In the Centers for Disease Control and Prevention's Guideline for Prescribing Opioids for Chronic Pain, Dowell et al. $^{8}$ advocate for sublingual (SL)

This article was externally peer reviewed.

Submitted 18 May 2020; revised 23 July 2020; accepted 24 July 2020.

From the Assistant Professor, Division of General Internal Medicine and Geriatrics, Section of Addiction Medicine, Oregon Health and Science University, Portland, OR; Associate Professor, Division of Hospital Medicine, Section of Addiction Medicine, Oregon Health and Science University, Portland, OR; and Associate Professor, Division of General Internal Medicine and Geriatrics, Section of Addiction Medicine, Oregon Health and Science University, Portland, OR.

Funding: No funding is reported.

Conflict of interest: No conflicts of interest are reported.

Correspondence author: Jonathan Robbins, MD, MS, Division of General Internal Medicine and Geriatrics, Mail Code L475, School of Medicine, Oregon Health and Science buprenorphine products for patients on prescription opioids who develop an opioid use disorder (OUD), but the authors did not explicitly recommend SLbuprenorphine as an alternative to tapering in patients with opioid dependence. Opioid dependence is the physiologic adaptation to chronic opioid use through the development of tolerance and withdrawal $^{6}$; OUD is characterized by craving, use despite consequences, loss of control, and compulsive use and is diagnosed using the Diagnostic and Statistical Manual of Mental Disorders, fifth edition (DSM-5). ${ }^{9}$ In 2019 Chou, Ballantyne, and Lembke ${ }^{10}$ argued for widespread use of SL buprenorphine as a complement to opioid tapering and to treat prescription opioid dependence, even in the absence of an OUD. Their rationale was that SL buprenorphine has important safety advantages, particularly in minimizing unintentional overdose and improved quality of life. ${ }^{10}$ This recommendation was further codified in the 2019 US Health and Human Services guidelines on opioid tapering and discontinuation. ${ }^{11}$

Buprenorphine is a partial agonist at the $\mu$ opioid receptor with high binding affinity and slow dissociation from the receptor. ${ }^{12}$ Low-dose transdermal and buccal buprenorphine products

University, 3181 SW Sam Jackson Park Road, Portland, OR 97239 (E-mail: robbijon@ohsu.edu). 
are Food and Drug Administration (FDA) approved for chronic pain and are typically used in opioid-naïve patients, whereas higher-dose SL buprenorphine is FDA approved for OUD only and considered off label for opioid dependence or pain. ${ }^{10}$ The partial agonist effect has significant safety benefits when compared with full agonist opioids; buprenorphine products are unlikely to cause respiratory arrest unless combined with other central nervous system depressants. ${ }^{13}$ Furthermore, buprenorphine has been hypothesized to reverse opioid hyperalgesia, a common and frustrating complication of long-term opioid therapy. ${ }^{14}$

Because buprenorphine has a higher binding affinity for the $\mu$ receptor than full agonist opioids, immediately starting buprenorphine in a patient taking full agonist will abruptly displace the full agonist from the $\mu$ receptor, leading to precipitated withdrawal. To avoid precipitated withdrawal, during conventional buprenorphine inductions, patients abstain from full agonist opioids until they experience moderate to severe opioid withdrawal. The conventional buprenorphine induction process is a barrier to treatment for both patients and providers. ${ }^{15}$ This is particularly true in patients with chronic pain, who fear both withdrawal and an increase in pain. ${ }^{16}$

Buprenorphine microdose inductions were first described by Hämmig in 2016 (the Bernese protocol) and involve repetitive, low-dose exposure to buprenorphine over several days, such that partial and full opioid agonists can be continued concurrently without precipitated withdrawal. ${ }^{17}$ Buprenorphine microinduction can improve care of patients with OUD by minimizing opioid withdrawal symptoms, reducing the dropout rate during induction, and decreasing fear of withdrawal. ${ }^{17}$ Microinductions have been shown to be safe and effective in inpatient and outpatient settings for patients with OUD. ${ }^{17-26}$ Less is known about SL buprenorphine microinductions for opioid-dependent patients on long-term opioid therapy (LTOT) for chronic, noncancer pain. ${ }^{27}$ Here we describe our experience using microdose buprenorphine inductions for patients with prescription opioid dependence in an urban, academic general internal medicine practice.

\section{Case Series}

From November 2019 to April 2020, providers completed 8 microdose buprenorphine inductions in opioid dependent patients on LTOT using a modified Bernese protocol ${ }^{17}$ (Table 1). We adapted a more gradual approach than the Bernese protocol, and taper rather than abruptly stop full agonist opioids, to improve patient acceptance of SL buprenorphine therapy ${ }^{27}$ (Table 2). Our protocol served as a general guide for the microinduction process, with minor variations in SL buprenorphine dosing and speed of full agonist taper, depending on clinical circumstances. We used the SL buprenorphine-naloxone combination product unless otherwise stated.

The average patient age was 63 years, all were white, and half were women. The insurance breakdown was 1 privately insured, 2 on Medicaid, 3 Medicare-Medicaid, and 2 Medicare only. The average morphine equivalent daily dose at the time of microinduction was $127 \mathrm{mg}$, although many patients had previously tapered from higher full agonist doses. Physical and mental comorbidities were common (Table 1). Two of the 8 patients met the DSM- 5 criteria for mild OUD at the time of microinduction; the remaining 6 had opioid dependence without an OUD.

All 8 patients tolerated SL buprenorphine microinduction without precipitated withdrawal. Two patients were unable to remain on buprenorphine-naloxone because of side effects that persisted after the induction phase and after tapering full agonist opioids. These included oversedation for 1 patient and persistent nausea for another. The side effects did not abate despite dose reduction or a trial of SL buprenorphine monoproduct. The 6 patients who successfully transitioned to SL buprenorphine reported stable to improved pain.

\section{Discussion}

Our institution has adopted a general dose limit for LTOT of 90 morphine equivalent daily dose for noncancer pain. Many patients are unable to taper to this dose because of profound disruption of the endogenous opioid system caused by years of exogenous high-dose opioids. ${ }^{28}$ For these patients with opioid dependence, transition to SL buprenorphine therapy is increasingly supported by federal guidelines and considered best practice. ${ }^{11}$ Patients and providers in our primary care practice have readily adopted the practice of SL buprenorphine microinductions. The microinduction protocol has eliminated concerns for precipitating opioid withdrawal 


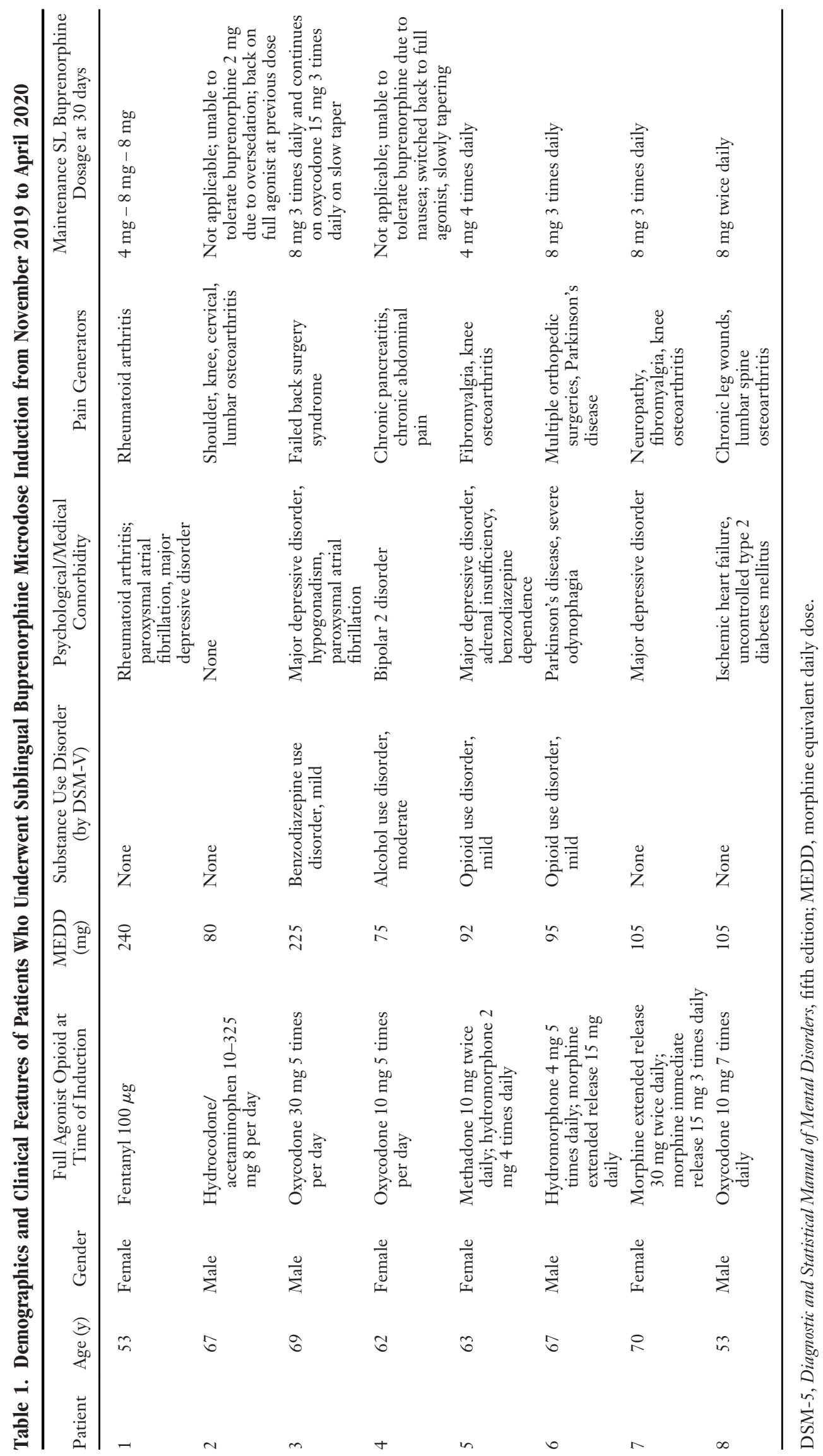


Table 2. Outpatient Microinduction Protocol Using Sublingual $2 \mathrm{mg}$ Buprenorphine/Naloxone Tablets or Films

\begin{tabular}{lll}
\hline Day & Bup/Nlx Dose and Frequency & $\begin{array}{c}\text { Full Agonist } \\
\text { Opioid }\end{array}$ \\
\hline 1 & $0.5 \mathrm{mg}$ daily (1/4 tablet or film) & No change \\
2 & $0.5 \mathrm{mg}$ BID & No change \\
3 & $1 \mathrm{mg}$ BID (half-tablet or film) & No change \\
4 & $2 \mathrm{mg}$ BID & No change \\
5 & $2 \mathrm{mg}$ TID & No change \\
6 & $4 \mathrm{mg}$ TID & No change \\
7 and beyond & Per provider discretion & Taper by 25\% \\
& & weekly \\
\hline
\end{tabular}

Bup, Buprenorphine; Nlx, naloxone; BID, twice a day; TID, twice a day.

during standard induction, which is a well-documented barrier to the use of buprenorphine. ${ }^{29}$

In our practice, patients on LTOT are often older with significant medical and psychiatric comorbidities and can be resistant to medication changes. Unlike other SL buprenorphine microinduction protocols for patients on LTOT, we choose to taper full agonist opioids by no faster than $25 \%$ a week after buprenorphine is initiated, rather than abruptly stopping opioids. ${ }^{27}$ At a dose of $16 \mathrm{mg}$ of SL buprenorphine, $90 \%$ of the $\mu$ receptors are occupied, and it is unlikely that discontinuing full agonist opioids after microinduction would lead to significant opioid withdrawal. ${ }^{30}$ Our approach recognizes the emotional distress and emotional pain that can accompany changes to opioid homeostasis, a concept referred to as hyperkatifeia. ${ }^{31} \mathrm{We}$ found that engaging in shared decision making about the speed of the full agonist opioid taper after initiating SL buprenorphine has increased acceptance of therapy and makes this transition more tolerable.

In the setting of the coronavirus 2019 (COVID19) pandemic, our practice minimized in-person visits and shifted clinical care to telephone or video visits, and some staff and providers have been redeployed to respiratory clinics. Conventional SL buprenorphine inductions can involve significant time commitments from providers and ancillary staff, including the active management of opioid withdrawal and daily titration of SL buprenorphine after home or office induction. ${ }^{29}$ Buprenorphine microinduction is well suited for telemedicine; inperson follow-up needs are minimal and can be addressed via phone or video visits. Opioid overdoses have increased during COVID-19, ${ }^{32}$ and there is an increasing call for low-barrier SL telebuprenorphine that has been facilitated by temporary changes in regulatory standards, which allow initiation of SL buprenorphine telephonically without an initial in-person visit. ${ }^{33-35}$

There are still several barriers to buprenorphine microinduction in our practice. Community pharmacists may not be familiar with microinduction protocols, and have refused to fill SL buprenorphine prescriptions for patients on full agonist opioids. Even after an explanatory phone call from our providers, this has undermined patient trust in the microinduction process. Practically, cutting SL $2 \mathrm{mg}$ buprenorphine tablets into quarters can be imprecise and inaccurate, although this has not interrupted the efficacy of the microinduction. Buprenorphine manufacturers could consider creating SL tablets and films at lower doses to accommodate the increasing number of opioid-dependent patients who will be transitioning to SL buprenorphine.

\section{Conclusions}

Microdose buprenorphine inductions have been well tolerated in opioid-dependent patients in a general internal medicine outpatient practice. Our case series adds to a growing body of evidence that SL buprenorphine microinduction is safe and feasible in diverse practice settings and populations. Despite the absence of randomized or case-controlled trials, we believe that microdose buprenorphine inductions should be offered to patients on LTOT who meet clinical criteria for a transition to SL buprenorphine therapy. This technique is low risk and can greatly reduce unnecessary suffering in the form of opioid withdrawal. Importantly, the FDA indication for high-dose SL buprenorphine products should expand to opioid dependence to reflect current guidelines. Buprenorphine microinductions are well suited for telemedicine and virtual care, which has important applications during the COVID-19 pandemic.

To see this article online, please go to: http://jabfm.org/content/ 34/Supplement/S141.full.

\section{References}

1. Wilson N, Kariisa M, Seth P, Smith H, Davis NL. Drug and opioid-involved overdose deaths-United States, 2017-2018. MMWR Morb Mortal Wkly Rep 2020;69:290-7. 
2. Strickler GK, Kreiner PW, Halpin JF, Doyle E, Paulozzi LJ. Opioid prescribing behaviors-prescription behavior surveillance system, 11 states, 2010-2016. MMWR Surveill Summ 2020;69:1-14.

3. Schieber LZ, Guy GP, Jr, Seth P, Losby JL. Variation in adult outpatient opioid prescription dispensing by age and sex-United States, 2008-2018. MMWR Morb Mortal Wkly Rep 2020;69:298-302.

4. Lembke A. Tapering long-term opioid therapy. Am Fam Physician 2020;101:49-52.

5. Lembke A, Humphreys K, Newmark J. Weighing the risks and benefits of chronic opioid therapy. Am Fam Physician 2016;93:982-90.

6. Manhapra A, Arias AJ, Ballantyne JC. The conundrum of opioid tapering in long-term opioid therapy for chronic pain: a commentary. Subst Abus 2018;39:152-61.

7. Darnall BD, Juurlink D, Kerns RD, et al. International stakeholder community of pain experts and leaders call for an urgent action on forced opioid tapering. Pain Med 2019;20:429-33.

8. Dowell D, Haegerich TM, Chou R. CDC guideline for prescribing opioids for chronic pain-United States, 2016. JAMA 2016;315:1624-45.

9. American Psychiatric Association, Diagnostic and statistical manual of mental disorders: DSM-5, fifth edition. ed. Arlington (VA): American Psychiatric Association; 2013.

10. Chou R, Ballantyne J, Lembke A. Rethinking opioid dose tapering, prescription opioid dependence, and indications for buprenorphine. Ann Intern Med 2019;171:427.

11. Health and Human Services. HHS guide for clinicians on the appropriate dosage reduction or discontinuation of long-term opioid analgesics. Washington (DC): Health and Human Services; 2019.

12. Foster B, Twycross R, Mihalyo M, Wilcock A. Buprenorphine. J Pain Symptom Manage 2013;45:939-49.

13. Sansone RA, Sansone LA. Buprenorphine treatment for narcotic addiction: not without risks. Innov Clin Neurosci 2015;12:32-6.

14. Khanna IK, Pillarisetti S. Buprenorphine-an attractive opioid with underutilized potential in treatment of chronic pain. J Pain Res 2015;8:859-70.

15. Gunderson EW. Buprenorphine induction: a major barrier for physician adoption of office-based opioid dependence treatment. J Addict Med 2011;5:304-5.

16. Darnall BD, Mackey SC, Lorig K, et al. Comparative effectiveness of cognitive behavioral therapy for chronic pain and chronic pain self-management within the context of voluntary patient-centered prescription opioid tapering: the EMPOWER study protocol. Pain Med 2019;21(8):1523-1531.

17. Hammig R, Kemter A, Strasser J, et al. Use of microdoses for induction of buprenorphine treatment with overlapping full opioid agonist use: the Bernese method. Subst Abuse Rehabil 2016;7:99-105.
18. Rozylo J, Mitchell K, Nikoo M, et al. Case report: successful induction of buprenorphine/naloxone using a microdosing schedule and assertive outreach. Addict Sci Clin Pract 2020;15:2.

19. Randhawa PA, Brar R, Nolan S. Buprenorphinenaloxone "microdosing": an alternative induction approach for the treatment of opioid use disorder in the wake of North America's increasingly potent illicit drug market. Cmaj 2020;192:E73. In:. Vol Canada.

20. Gudin J, Fudin J. A narrative pharmacological review of buprenorphine: a unique opioid for the treatment of chronic pain. Pain Ther 2020;9: 41-54.

21. De Aquino JP, Fairgrieve C, Klaire S, GarciaVassallo G. Rapid transition from methadone to buprenorphine utilizing a micro-dosing protocol in the outpatient Veteran Affairs setting. J Addict Med 2020;14(5):e271-e273.

22. Terasaki D, Smith C, Calcaterra SL. Transitioning hospitalized patients with opioid use disorder from methadone to buprenorphine without a period of opioid abstinence using a microdosing protocol. Pharmacotherapy 2019;39:1023-9.

23. Raheemullah A, Lembke A. Initiating opioid agonist treatment for opioid use disorder in the inpatient setting: a teachable moment. JAMA Intern Med 2019;179:427-8.

24. Martin L, Lennox R, Regenstreif L, O'shea T. Case report: "striving to skip the withdrawal" using buprenorphine-naloxone microdosing for hospitalized patients. Cana J Addict 2019;10:35-40.

25. Lee DS, Hann JE, Klaire SS, Nikoo M, Negraeff MD, Rezazadeh-Azar P. Rapid induction of buprenorphine/naloxone for chronic pain using a microdosing regimen: a case report. A A Pract 2019;14(2): 44-47.

26. Klaire S, Zivanovic R, Barbic SP, Sandhu R, Mathew $\mathrm{N}$, Azar P. Rapid micro-induction of buprenorphine/ naloxone for opioid use disorder in an inpatient setting: a case series. Am J Addict 2019;28:262-5.

27. Becker WC, Frank JW, Edens EL. A switching from high-dose, long-term opioids to buprenorphine: a case series. Ann Intern Med 2020;173 (1):70-71.

28. Koob GF. Neurobiology of opioid addiction: opponent process, hyperkatifeia, and negative reinforcement. Biol Psychiatry 2020;87:44-53.

29. Walley AY, Alperen JK, Cheng DM, et al. Officebased management of opioid dependence with buprenorphine: clinical practices and barriers. J Gen Intern Med 2008;23:1393-8. Vol.

30. Zubieta J, Greenwald MK, Lombardi U, et al. Buprenorphine-induced changes in mu-opioid receptor availability in male heroin-dependent volunteers: a preliminary study. Neuropsychopharmacology 2000; 23:326-34. 
31. Davis MP, Digwood G, Mehta Z, McPherson ML. Tapering opioids: a comprehensive qualitative review. Ann Palliat Med 2020;9:586-610.

32. Slavova S, Rock P, Bush HM, Quesinberry D, Walsh SL. Signal of increased opioid overdose during COVID-19 from emergency medical services data. Drug Alcohol Depend 2020;214:108176.

33. Harris M, Johnson S, Mackin S, Saitz R, Walley AY, Taylor JL. Low barrier tele-buprenorphine in the time of COVID-19: a case report. J Addict Med 2020;14(4):e136-e138.

34. Dunlop A, Lokuge B, Masters D, et al. Challenges in maintaining treatment services for people who use drugs during the COVID-19 pandemic. Harm Reduct J 2020;17:26.

35. Becker WC, Fiellin DA. When epidemics collide: coronavirus disease 2019 (COVID-19) and the opioid crisis. Ann Intern Med 2020;173:59-60. 